\title{
A Priority-Aware Protection Technique for Quality of Service Enabled WDM Networks
}

\author{
W. Fawaz ${ }^{1}$, F. Martignon ${ }^{2}$, K. Chen ${ }^{1}$, and G. Pujolle ${ }^{3}$ \\ 1 University of Paris 13 - L2TI Lab, 99, Avenue Jean-Baptiste Clement, \\ 93430 Villetaneuse, France \\ m.fawaz@isep.fr \\ chen@galilee.univ-paris13.fr \\ 2 Dipartimento Ingegneria Gestionale e dell'Informazione, \\ University of Bergamo, Italy \\ fabio.martignon@unibg. it \\ 3 University of Paris 6, LIP6 Laboratory, \\ 8 rue du Capitaine Scott, 75015, Paris, France \\ guy.pujolle@lip6.fr
}

\begin{abstract}
Connection availability is considered as a critical metric, when providing differentiated services in WDM mesh networks. Indeed, one of the major concerns of optical network operators is related to improving the availability of services provided to their highest-class clients. Achieving this objective is possible through the use of the different classical protection schemes, namely the so-called dedicated and shared protection schemes. However, the majority of the work concerning protection schemes has considered the primary connections as equally important when contending for the use of the backup resources.

As a main contribution in this paper, we therefore propose an improvement of the existing protection schemes through the introduction of relative priorities among the different primary connections contending for the access to the protection path. To evaluate numerically the benefits of the service differentiation feature introduced in our proposal, we first develop a mathematical model based on which we derive explicit expressions for the average connections availabilities that result from both the classical protection schemes and the proposed priority-aware one. Through this model, we show how the availability of the highest-class clients is improved when deploying the proposed priority-aware protection scheme.

Finally, with the same objective in mind, we develop a simulation study, where a given set of connection demands with predefined availability requirements is provisioned, using different protection strategies. Through this study, we show that the priority-aware protection strategy satisfies service-availability requirements in a cost-effective manner compared with the classical protection schemes.
\end{abstract}

\section{Introduction}

The revolutionary Wavelength-Division multiplexing (WDM) technology increases the transmission capacity of fiber links by several orders of magnitude. 
As WDM keeps on evolving, fibers are witnessing a huge increase regarding their carriage capacity, which has already reached the order of terabits per second. Therefore, the failure of a network component (e.g., a fiber link, an optical cross connect, an amplifier, a transceiver, etc) can weigh heavily on optical carrier operators due to the consequent huge loss in data and revenue. To get an estimate of the different optical components failure characteristics, Table 1 presents the mean failure rates and failure repair times of various optical network components according to Bellcore (now Telecordia) [1, where Failure-In-Time (FIT) denotes the average number of failures in $10^{9}$ hours, Tx denotes optical transmitters, $\mathrm{Rx}$ denotes optical receivers, and MTTR stands for Mean Time To Repair.

Table 1. Failure rates and repair times (Telecordia [1])

\begin{tabular}{c|c}
\hline Metric & Telecordia Statistics \\
\hline Equipment MTTR & $2 \mathrm{~h}$ \\
\hline Cable-cut MTTR & $12 \mathrm{~h}$ \\
\hline Cable-cut rate & $501142 \mathrm{FIT} / 1000$ sheat miles \\
\hline Tx failure rate & $10867 \mathrm{FIT}$ \\
\hline Rx failure rate & $4311 \mathrm{FIT}$ \\
\hline
\end{tabular}

Two main conclusions may be drawn based on these statistics: the frequency of failure occurrence in optical networks is not negligible; moreover, cable cut is the dominant failure scenario, compared to Tx and Rx failures, for lengths in the order of hundreds of kilometers, normally found in backbone optical networks. With the frequent occurrence of fiber cuts and the tremendous loss that a failure may cause, network survivability, together with its impact on network design, becomes a critical concern for operators who strive to keep up with the competition for broadband traffic transport. Moreover, as WDM networks migrate from ring to mesh topology, planning a survivable WDM mesh network has been the subject of extensive studies [3, 4, 5, leading to the definition of various resilience approaches. Mainly, there are two types of fault recovery mechanisms: protection [6] and restoration schemes [7]. In this paper we focus our study on protection schemes, dealing mainly with the impact these schemes have on the customer-perceived service quality which is an emerging topic and of special interest today. We believe that protection, a proactive procedure, is a key strategy to ensure fiber network survivability. To the best of our knowledge what still lacks in existing literature is a systematic methodology to efficiently select a cost-effective protection scheme for each connection, while satisfying its quality of service (QoS) requirements. Usually, by means of service contracts called Service Level Agreements (SLA), a client subscribes to optical network services from the optical operator with a certain guaranteed QoS level. Within the SLA, Service Level Specifications (SLS) 8] quantify the quality of service provided to the customer. A certain number of SLSs indicate the reliability constraints needed by the subscribed service. Reliability parameters presented in the literature include mainly service availability, and restoration time. Our interest will be 
directed to service availability since the problem of how connection availability is affected by network failures is currently attracting more research interest.

As a first main contribution in this paper, we propose an extension for the so-called shared protection scheme contributing to the design of new quality of service-aware protection schemes. In order to gauge the benefits of our proposal, the impact of such an approach on the customer-perceived service availability needs to be studied and to be compared with the classical protection approaches. Moreover, to assess the efficiency of the proposed scheme in comparison to the classical protection schemes, we need to evaluate the cost in terms of resources (i.e., number of wavelengths needed for instance) induced from the deployment of both the priority-aware scheme and the classical schemes in a real network. Therefore, we first present a mathematical model for both the classical sharedprotection schemes and the proposed priority-aware scheme. We derive explicit analytic expressions for the average availability resulting from the deployment of such schemes. By solving these models we then evaluate numerically the benefits of the service differentiation feature introduced in our scheme. Finally, we exhibit the cost-effectiveness of our proposed approach regarding resource consumption (i.e., wavelengths) in a sample optical network topology using a simulation study. In this regard, a given set of randomly generated connection demands with predefined availability requirements are routed in the network using several provisioning schemes (i.e., using unprotected, dedicated, shared, and priority-aware shared protection schemes). The performances of these provisioning schemes are compared in terms of resources needed in the network, and in terms of the connections availability satisfaction rate. Our results show that the proposed protection approach achieves a high satisfaction rate while greatly economising resource usage. The paper is structured as follows: in Section II we revise the related works presented in the litterature, pointing out our position relative to these works; in Section III we propose and describe the priority-aware shared protection scheme; in Section IV we introduce a mathematical model to evaluate the impact of the protection schemes analyzed in this paper on the connection availability; in Section V we present numerical results based on the mathematical study to evaluate the benefits of the service differentiation feature introduced in our scheme. In section VI, the simulation study is developed with the corresponding results. Finally, Section VII concludes this paper and proposes future issues.

\section{Priority-Aware Shared-Protection Scheme}

This Section introduces the proposed protection scheme that extends the existing shared-protection schemes through the introduction of relative priorities among different primary connections contending for the backup paths. Let us consider $N$ working paths $\left(w_{i}, i=1, \ldots, N\right)$ with the same source and destination sharing $M$ backup paths $\left(b_{i}, i=1, \ldots, M\right)$, i.e. an M:N protections scheme, as depicted in Figure 1. Both work paths and backup paths can be in failure. When a failure occurs, the repair process is started. 


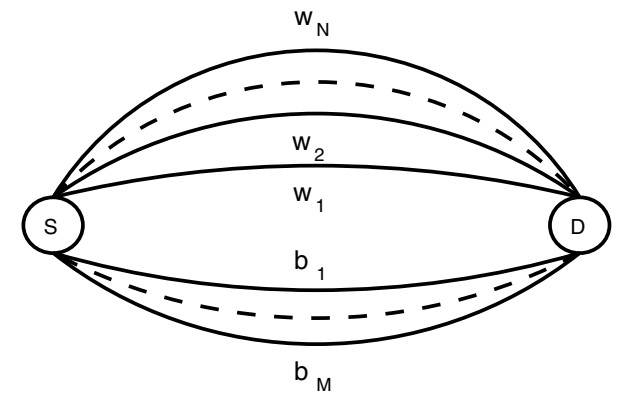

Fig. 1. $\mathrm{N}$ working paths sharing $\mathrm{M}$ backup paths between a source node $\mathrm{S}$ and a destination node $\mathrm{D}$

In the classical shared-protection scheme, when several subsequent failures happen in the network, all connections are considered of equal importance when contending for backup resources. As such, the first failed connection gains access to the backup path. On the other hand, in our proposed scheme these connections are divided into $K$ sets of reliability classes, $C_{1}, \ldots, C_{K}$, with $N_{i}$ connections belonging to class $C_{i}$ for $i=1$ to $K$, and $\sum_{i=1}^{K} N_{i}=N$. Connections belonging to class $C_{1}$ have the highest priority, while those belonging to $C_{K}$ have the lowest priority. When the working path of a connection $t$ belonging to class $C_{i}$ breaks down, the first available backup path, if any, is assigned to protect connection $t$ and restoration is ensured by switching $t$ to the backup path. Meanwhile, repair actions are performed on the primary path to restore it to be as good as new. Once repairing the primary path is achieved, the restored connection is switched back to its primary path. On the contrary, if at the moment $t$ fails all the backup paths are already occupied protecting other connections, a check is made to verify the existence of protected connections belonging to classes of lower priority than $t$, i.e. to classes comprised between $i+1$ and $K$. If several such connections exist, the one having the lowest priority is immediately preempted by connection $t$. The preempted connection thus becomes unavailable, waiting for a backup path to be freed or for its working path to be repaired. Finally, if neither of the two above situations is verified, connection $t$ becomes unavailable.

\section{The Mathematical Model}

In this Section, we present a mathematical model for both the classical 1:N shared protection scheme and the corresponding priority-aware extension discussed previously. Solving this model, we derive explicit expressions for the average availability of a connection resulting from deploying the aforementioned protection strategies. It is important to note that the dedicated protection case can be viewed as a special case of the shared protection scheme with $\mathrm{N}=1$. As we are interested in the availability of a connection, we need to define it first. The availability of a connection is defined as the probability that such connec- 
tion is "up" at any given time [9], and can be expressed as the proportion of time the connection is up during its entire service. If a connection is carried by a single unprotected path, its availability is equal to the path availability. The availability of a protected connection is determined by both the primary and the backup paths. In other words, a protected connection $t$ is said to be available when either no failure affects its primary path or it is recovered by the backup path in case of failure along the primary path. Connection $t$ becomes unavailable in the following two cases:

- one failure occurs on the primary path of $t$ and a second failure occurs on its backup path;

- if $t$ shares the backup path with connection $t^{\prime}$, then $t$ will be unavailable if both $t$ and $t^{\prime}$ fail but the shared backup path is taken by $t^{\prime}$. In the priorityaware scheme, this happens if $t^{\prime}$ has higher priority than $t$.

\subsection{Model Definition and Resolution for the Classic Shared-Protection Scheme}

Let us consider $N$ working paths that share the same backup path, i.e. a 1:N shared-protection scheme. Let $\lambda_{i}, i=1, \ldots, N+1$ be the mean failure rate of the $i$-th path and $\mu_{i}$ be the mean recovery rate of the $i$-th path; $\frac{1}{\lambda_{i}}$ and $\frac{1}{\mu_{i}}$ hence represent the Mean Time To Failure and Mean Time To Repair of the $i$-th path, respectively. Based on the above assumptions, all the path failures are statistically independent, and interfailure and repair times are exponentially distributed. To gain insight into the behavior of the system and according to existing literature [10,11, we will consider a case of special interest in which all the paths (working as well as backup ones) have identical failure and recovery rates, i.e. $\lambda_{i}=\lambda$ and $\mu_{i}=\mu, \forall i=1, \ldots, N+1$. Let us define $\rho=\frac{\lambda}{\mu}$. We have here a classical problem of reliability, with 1 redundant unit for $\mathrm{N}$ working units. Here a unit is an optical path. The steady-state availability $A_{i}$ of a single path $i$, viz. the limiting $(\tau \rightarrow \infty)$ probability of finding the path successfully operating at time $t$, can be calculated as follows:

$$
A_{i}=\frac{M T T F}{M T T F+M T T R}=\frac{1 / \lambda}{1 / \lambda+1 / \mu}=\frac{1}{1+\rho}
$$

$\overline{A_{i}}=1-A_{i}$ represents the unavailability of path $i$.

Let $F(\tau)$ be the number of failed paths at time $t$. Because of the assumptions, $F(\tau) ; \tau \geq 0$ forms a continuous and stationary Markov process, with $F(0)=0$. Let $p(n)$ be the steady state probability that $F(\tau)=n$ in stationary regime. The transition diagram is given in Fig. 2.

After some classical calculus we can express the steady state probability $p(n)$ of the Markov chain as follows [10, 12]:

$$
p(n)=C_{N+1}^{n} \bar{A}^{n} A^{N+1-n}=\frac{(N+1) !}{n !(N+1-n) !(1+\rho)^{N+1}}
$$



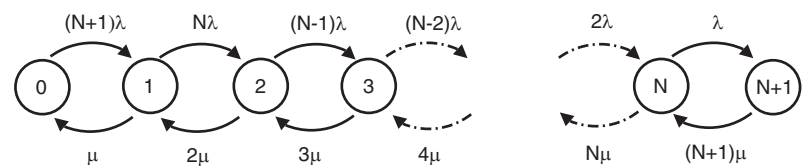

Fig. 2. Transition Diagram

where $C_{N+1}^{n}$ represents the number of all combinations of $n$ failed paths out of $N+1$, and $A$ is given by equation (1). In other words, the number of failed paths follows a binomial distribution with parameters $N+1$ and $\bar{A}$.

Note that $p(n)$ represents the proportion of time in which there are $n$ failures in the network. When the total number of path failures $n$ is greater than or equal to one, we can distinguish two cases:

1. the backup path is among the failed paths and the remaining $n-1$ connections cannot be restored;

2. all the $n$ failed paths are primary paths, and as such, only one connection is restored by the backup path while the remaining $n-1$ are not.

Therefore, under such conditions there will always be exactly $n-1$ unavailable connections. For $n \geq 2$ at least one connection will be unavailable, while when the number of failures $n$ is equal to 1 , there will be no unavailable connections. From this classical result, we are now interested in calculating the average unavailability of a specific connection $t$ among the $N$ shared-protected ones. The average unavailability of $t$ is the proportion of time such connection is unavailable for all possible numbers of failures $n, 2 \leq n \leq N+1$. Let us define $Y(n)$ the event of $t$ being unavailable under state $n$. The probability of having our reference connection $t$ unavailable when there are $n$ failed paths is equal to $p(n) P(Y(n))$. As $p(n)$ has already been calculated in equation (2), what remains is to calculate $P(Y(n))$. To do so, we have to consider all the events that may lead to the connection $t$ becoming unavailable under state $n$. These events are the following: $W(n)$ : both the primary path of connection $t$ and the backup path are failed; $Z(n)$ : connection $t$ 's primary path is failed but the backup path is available.

Building on this information and according to the theorem of total probability, $P(Y(n))$ can be calculated as follows

$$
P(Y(n))=P(Y(n) \mid W(n)) P(W(n))+P(Y(n) \mid Z(n)) P(Z(n))
$$

where $P(Y(n) \mid W(n))$ and $P(Y(n) \mid Z(n))$ are, respectively, the conditional probabilities of having our reference connection $t$ unavailable, given that events $W(n)$ and $Z(n)$ occurred. $P(Y(n) \mid W(n))=1$ as the backup path in this case is failed and no restoration is possible; $P(Y(n) \mid Z(n))=\frac{n-1}{n}$ as only one of the $n$ primary paths under failure in this case can be restored.

The probability of the event $W(n)$ is:

$$
P(W(n))=\frac{C_{N-1}^{n-2}}{C_{N+1}^{n}}=\frac{n(n-1)}{N(N+1)}
$$


where the numerator indicates all possible combinations where the primary path of connection $t$ and the backup path are among the failures. The denominator indicates all possible combinations of $n$ failed paths out of $N+1$.

The probability of the event $Z(n)$ is:

$$
P(Z(n))=\frac{C_{N-1}^{n-1}}{C_{N+1}^{n}}=\frac{n(N+1-n)}{N(N+1)}
$$

where the numerator indicates all possible combinations where the primary path of the connection $t$ is among the failures while the backup is not.

Then, based on the above equations, the probability $\mathrm{P}(\mathrm{Y}(\mathrm{n}))$ that the observed connection $t$ is unavailable under state $n$ is equal to:

$$
P(Y(n))=\frac{n-1}{N}, 2 \leq n \leq N+1
$$

It can be seen that this equation is also valid for the case $n=1$, for which $P(Y(n))=0$, since in this case all connections will be available, as stated before. Based on the theorem of total probability, the unavailability of a connection in the case of $1: \mathrm{N}$ protection is given by the following formula:

$$
U(N, \lambda, \mu)=\sum_{n=2}^{N+1} p(n) \cdot P(Y(n))=\sum_{n=2}^{N+1} p(n) \cdot \frac{n-1}{N}
$$

and, substituting the expression (11) for $p(n)$ we obtain:

$$
U(N, \lambda, \mu)=\frac{1}{N} \cdot \sum_{n=2}^{N+1} \frac{(n-1) \cdot C_{N+1}^{n} \cdot \rho^{n}}{(1+\rho)^{N+1}}
$$

The average availability for a connection is simply equal to $1-U(N, \lambda, \mu)$.

\subsection{Model Definition and Resolution for the Priority-Aware Scheme}

Let us consider the priority-aware shared-protection system proposed in Section III, where $N$ connections are divided into two sets of reliability classes, $C_{1}$ and $C_{2}$, with $N_{1}$ and $N_{2}$ connections belonging to class $C_{1}$ and $C_{2}$, respectively, and $N_{1}+N_{2}=N$. Connections of class $C_{1}$ have higher priority than connections belonging to $C_{2}$. In the following we derive the analytic expressions for the availability for each connection according to its priority class. We will begin by considering higher-priority connections. First of all, the $N_{1}$ connections having the highest priority can preempt instantaneously all the other connections belonging to the lower-priority class in the utilization of the backup path. Consequently, the analysis of the proposed scheme with regard to the high-priority connections is equivalent to the study of a classic $1: N_{1}$ shared-protection scheme. Therefore, we can derive straightforwardly the average unavailability $U_{1}$ of highpriority class connections based on equation (8) by simply substituting $N$ with $N_{1}$. 


$$
U_{1}\left(N_{1}, \lambda, \mu\right)=\frac{1}{N_{1}} \cdot \sum_{n=2}^{N_{1}+1} \frac{(n-1) \cdot C_{N_{1}+1}^{n} \cdot \rho^{n}}{(1+\rho)^{N_{1}+1}}
$$

When a low-priority connection fails, it becomes unavailable if any of the following mutually exclusive conditions is verified:

1. the protection path has already failed;

2. the protection path is up but there is at least one high-priority connection among the failures;

3. the protection path is up, no high-priority connections are among failures, there is however another low-priority connection occupying the protection path.

Let $E_{i}$ be the event of having condition $i$ verified, $i=1,2,3$. Therefore, to study the unavailability $U_{2}$ of a low-priority connection, we consider the process $Q(\tau)$ whose general state is a triplet $\left(n_{1}, n_{2}, b\right)$, where $n_{1}$ and $n_{2}$ indicate, respectively, the number of failed high and low-priority connections at time $\tau$, and $b$ is a flag set to 1 if the backup path is down and to 0 if it is up. $Q(\tau)$ is a continuous and stationary Markov process, with a limiting probability for each state given by

$$
P\left(n_{1}, n_{2}, b\right)=P\left(n_{1}\right) P\left(n_{2}\right) P(b)
$$

where $P\left(n_{1}\right)$, the probability of having $n_{1}$ failed high-priority connections and $P\left(n_{2}\right)$, the probability of having $n_{2}$ failed low-priority connections, are respectively equal to:

$$
\begin{aligned}
& P\left(n_{1}\right)=C_{N_{1}}^{n_{1}} \bar{A}^{n_{1}} A^{N_{1}-n_{1}} \\
& P\left(n_{2}\right)=C_{N_{2}}^{n_{2}} \bar{A}^{n_{2}} A^{N_{2}-n_{2}}
\end{aligned}
$$

and $A$ is given by equation (11). $P(b)$ is the probability of having $b$ backup path failures. In other words, when $b=0$, there is no failure affecting the backup path, whereas if $b=1$ the backup path is down. The expression of $P(b)$ is:

$$
P(b)=\bar{A}^{b} A^{1-b}
$$

The events $\left(E_{i}, i=1,2,3\right)$, leading to the unavailability of a low-priority connection, are verified according to the values of $n_{1}, n_{2}$ and $b$. So, $b=1$ leads to $E_{1}$, meaning that the protection path has failed; on the other hand, $b=0$ and $n_{1} \geq 1$ lead to event $E_{2}$; finally, $b=0, n_{1}=0$ and $n_{2} \geq 2$ produce event $E_{3}$. Under state $\left(n_{1}, n_{2}, b\right)$, a specific low-priority connection $t$ is unavailable when it fails and one of the events $E_{1}-E_{3}$ is produced. Based on this observation, $U_{2}$ is given by:

$$
U_{2}=\sum_{\forall\left(n_{1}, n_{2}, b\right)} P\left(t \text { fails in state }\left(n_{1}, n_{2}, b\right)\right) \cdot P\left(n_{1}, n_{2}, b\right) \cdot P\left(E_{1} \cup E_{2} \cup E_{3}\right)
$$


where:

$$
P\left(t \text { fails in state }\left(n_{1}, n_{2}, b\right)\right)=\frac{C_{N_{2}-1}^{n_{2}-1}}{C_{N_{2}}^{n_{2}}}
$$

and $P\left(E_{1} \cup E_{2} \cup E_{3}\right)$ can be obtained with classical manipulations. It follows that $U_{2}$ is equal to:

$$
\begin{aligned}
U_{2} & =\sum_{i=2}^{N_{2}+1} C_{N_{2}-1}^{i-2} \bar{A}^{i} A^{N_{2}-i+1}+\sum_{i=1}^{N_{2}} C_{N_{2}-1}^{i-1} \bar{A}^{i} A^{N_{2}-i+1} \cdot\left(1-A^{N_{1}}\right)+ \\
& +\sum_{i=2}^{N_{2}} C_{N_{2}-1}^{i-1} \bar{A}^{i} A^{N_{2}-i+1} \cdot A^{N_{1}} \cdot \frac{(i-1)}{i}
\end{aligned}
$$

\section{Numerical Results}

In this Section we gauge the benefits the proposed priority-aware protection scheme through numerical results induced from the previous mathematical study. For the sake of simplicity, we consider a scenario consisting of 3 primary connections sharing one backup path. We first consider a priority-aware protection scheme, with one high-priority and two low-priority primary connections.The availability of each class is calculated for different connections' lengths based on equations (9) and (14), and is reported in Figure 3. Then, a classical shared protection scheme is applied to this scenario, and the availability of a connection is evaluated using equation (8). The corresponding results are reported again in Figure 3 for comparison purposes. It is important to state that the Mean Time To Repair $\left(\frac{1}{\mu}\right)$ of all the paths is considered equal to 12 hours (see Table I).

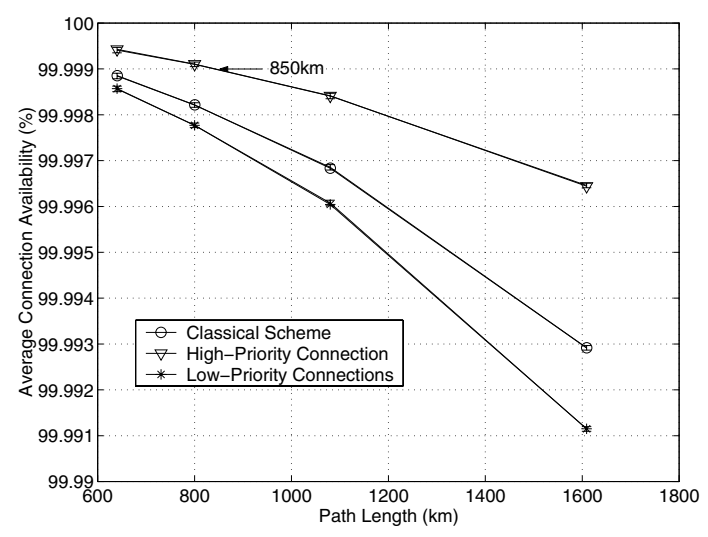

Fig. 3. Average availability for the classical and the priority-aware 1:3 sharedprotection scheme 
Based on Figure 3 we can observe that the high-priority connection protected using the priority-aware scheme is more available than the connections protected by the classical shared scheme. The observed availability results can be interpreted from a Quality of Service level perspective using the following reasoning. According to [8] a Platinum client requests an availability of $99.999 \%$ (i.e. at most 5 minutes of unavailability per year), whereas a Gold client requires an availability of $99.99 \%$ per year. With regard to this QoS terminology, the high and the low-priority classes can be mapped into Platinum and Gold QoS levels [8] or to lower QoS classes according to the connection's length.

In fact, as shown in Figure 3, the availability of the high-priority connection drops below $99.999 \%$ when the connection length exceeds $850 \mathrm{~km}$, while in the classical scheme this target availability is never achieved. This proves that by deploying the proposed scheme, Platinum connections provisioning becomes possible in the network even for long communications which are encountered typically in backbone optical networks. Moreover, the QoS level of the Gold connections is still maintained.

\section{$5 \quad$ Simulation Study}

For illustration purposes and following the guidelines presented in 13 , in our simulation we consider the network topology of Figure 4, availability of fibers is a pre-assigned value which could be $99.8 \%, 99.9 \%, 99.95 \%$, or $99.995 \%$ according to their length. A traffic matrix of connection requests among all node pairs (i.e., Total number of connections $=24 \times 23$ connections) is randomly generated. The availability requirements of the connection requests are uniformly distributed between two classes: $99.9 \%$, or $99.99 \%$, which are referred to as Silver and Gold classes respectively. The traffic matrix is routed in the network according to different provisioning schemes which adopt distinct protection strategies. The availability for each provisioned connection is then calculated and compared to its required availability. Based on this, the Availability Satisfaction Rate resulting from each provisioning scheme for both Gold $\left(A S R_{G}\right)$ and Silver $\left(A S R_{S}\right)$

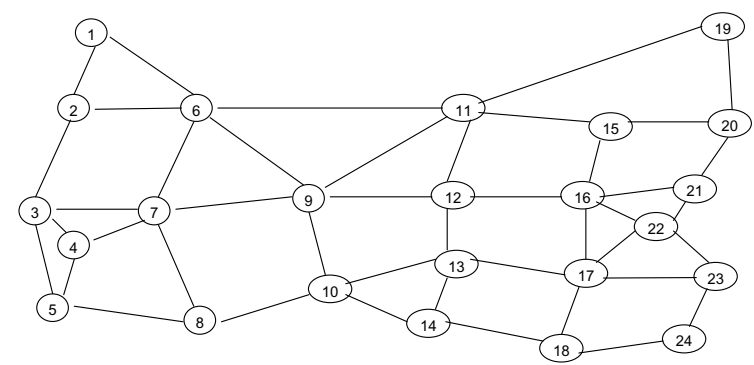

Fig. 4. A sample network topology 
Table 2. Results from Four Provisioning Schemes

\begin{tabular}{||c|c|c|c|c||}
\hline Scheme & $A S R_{G}$ & $A S R_{S}$ & $W$ & $W_{\text {Total }}$ \\
\hline \hline Scheme I & $5 \%$ & $20 \%$ & 94 & 3352 \\
\hline Scheme II & $100 \%$ & $100 \%$ & 180 & 7961 \\
\hline Scheme III & $94 \%$ & $100 \%$ & 150 & 6182 \\
\hline Scheme IV & $100 \%$ & $100 \%$ & 150 & 6182 \\
\hline
\end{tabular}

connections is computed and reported in Table 2]. Moreover in Table 2, the performance of the different provisioning schemes is stipulated in terms of the number of wavelength channels needed $(W)$, and the total number of wavelength links $\left(W_{\text {Total }}\right)$. $W$ denotes the number of wavelength channels on the most congested fiber. On the other hand, $W_{\text {Total }}$ denotes the total number of consumed wavelengths in the whole network.

We compare the performance of four different provisioning schemes:

Scheme I (Without Protection) where all connections are routed using a simple Dijkstra algorithm applied to the hop number without any protection, and without any connection-availability consideration; Scheme II (Dedicated Protection) where all connections are provisioned with dedicated-path protection (i.e.,1:1 protection); Scheme III (Classical Shared-Path Protection) where all connections are provisioned with the classical shared-path protection; and Scheme IV (Priority-Aware Shared Protection) where all connections are provisioned according to the proposed priority-aware shared-path protection.

From Table 2, one can observe that Scheme I consumes the least amount of resources compared with the other schemes. But in Scheme I, only $5 \%$ of Gold and $20 \%$ of Silver connections can meet their required availabilities. This is because the primary path in Scheme I is calculated according to the minimum number of hops but it may not be reliable enough. By deploying a dedicated protection as in Scheme II, the Gold and Silver connection Availability Satisfaction rates $\left(A S R_{G}, A S R_{S}\right)$ reach $100 \%$; however, a large amount of resources is consumed. By providing a classical shared protection scheme as in Scheme III, an optimization of resource usage is achieved while realizing high $A S R \mathrm{~s}$ but the Availability Satisfaction Rate of Gold connections drops below 100\%. Finally, when deploying the priority-aware protection scheme proposed in this paper (Scheme IV), the Availability Satisfaction Rates for both Gold and Silver connections $\left(A S R_{G}, A S R_{S}\right)$ attain $100 \%$ while optimizing resource usage.

\section{Conclusions}

In this paper we have proposed an improvement of the existing shared protection schemes through the introduction of relative priorities among the different primary connections contending for the access to the protection path. We presented a detailed mathematical model for both the classical shared-protection schemes and for the proposed priority-aware scheme. We derived explicit analytic expressions for the average availability resulting from the deployment of 
such schemes. Through this study, it has been proven that service differentiation is better achieved through the use of our proposed protection scheme.

Finally, we developed a simulation study where it has been shown that the proposed scheme achieves high Availability Satisfaction Rates while realizing cost-effectiveness in terms of resource usage in the network.

\section{References}

1. Jing Zhang and B.Mukherjee. A Review of Fault Management in WDM Mesh Networks: Basic Concepts and Research Challenges. In IEEE Network, pages 4148 vol.18(2), March-April 2004.

2. R.Ramaswami. Optical Fiber Communication: From Transmission to Networking. In IEEE Communications Magazine, pages 138-147 vol.40(5), May 2002.

3. S.Ramamurthy, L.Sahasrabuddhe, and B.Mukherjee. Survivable WDM Mesh Networks. In Journal of Lightwave Technology, pages 870-883, vol. 21(4), April 2003.

4. G.Mohan, S.R.Murthy, and A.K.Somani. Efficient Algorithms for Routing Dependable Connections in WDM Optical Networks. In IEEE/ACM Transactions on Networking, pages 553-566 vol.9, Oct. 2001.

5. G.Ellinas, A.Hailemariam, and T.E.Stern. Protection Cycles in Mesh WDM Networks. In IEEE Journal on Selected Areas in Communications, pages 1924-1937 vol.18, October 2001.

6. S.Ramamurthy and B.Mukherjee. Survivable WDM Mesh Networks, Part I Protection. In Proceedings of INFOCOM'99, pages 744-751, March 1999.

7. S.Ramamurthy and B.Mukherjee. Survivable WDM Mesh Networks, Part II Restoration. In Proceedings of IEEE International Conference on Communications (ICC '99), pages 2023-2030, June 1999.

8. W.Fawaz, B.Daheb, O.Audouin, B.Berde, M.Vigoureux, M.Du-Pond, and G.Pujolle. Service Level Agreement and Provisioning in Optical Networks. In IEEE Communications Magazine, June 2004.

9. M.To and P.Neusy. Unavailability Analysis of Long-Haul Networks. In IEEE Journal on Selected Areas in Communications, pages 100-109 vol.12(1), 1994.

10. J.E.Angus. On Computing mtbf for a k-out-of-n:g Repairable System. In IEEE Transactions on Reliability, volume Vol 37(3), pages 312-313, August 1988.

11. D.Lee, L.Libman, and A.Orda. Path Protection and Blocking Probability Minimization in Optical Networks. In Proceedings of INFOCOM'04, 7-11 March 2004.

12. D.Mitra. Stochastic Theory of a Fluid Model of Producers and Consumers Coupled by a Buffer. In Advances in Applied Probability, pages 646-676 vol.20, 1988.

13. H.Zang J.Zhang, K.Zhu and B.Mukherjee. A New Provisioning Framework to Provide Availability-Guaranteed Service in WDM Mesh Networks. In Proceedings of ICC 2003, pages 1484-1488, May 2003. 